\title{
Enhanced bound-state formation in two dimensions via stripelike hopping anisotropies
}

\author{
Saurabh Basu and R. J. Gooding \\ Department of Physics, Queens University, Kingston, Ontario, Canada K7L 3N6 \\ P. W. Leung \\ Department of Physics, Hong Kong University of Science and Technology, Clear Water Bay, Hong Kong
}

(Received 5 October 2000; published 20 February 2001)

\begin{abstract}
We investigate two-electron bound-state formation in a square two-dimensional $t-J-U$ model with hopping anisotropies for zero-electron density; these anisotropies are introduced to mimic the hopping energies similar to those expected in the stripelike arrangements of holes and spins found in various transition metal oxides. We provide complete analytical solutions to this problem, and thus demonstrate that bound-state formation occurs at a critical exchange coupling, $J_{c}$, that decreases to zero in the limit of extreme hopping anisotropy $t_{y} / t_{x} \rightarrow 0$. This behavior is found to be qualitatively identical to that of two electrons on a two-leg ladder in the limit of $t_{\text {interchain }} / t_{\text {intrachain }} \rightarrow 0$. Using the latter result as guidance, we have determined that this bound state corresponds to one electron moving along one chain, with the second electron moving along the opposite chain, similar to two electrons confined to move along parallel neighboring metallic stripes. Further, we have found that similar behavior is found in systems doped with two holes away from half filling.
\end{abstract}

DOI: 10.1103/PhysRevB.63.100506

PACS number(s): 74.20.Mn, 71.27.+a, 71.10.Fd

The experimental verification for the existence of stripes in layered transition-metal oxides, such as the high- $T_{c}$ superconductors, is now robust, ${ }^{1}$ and yet an important question remains: Does the presence of such rivers of charge help, hinder, or even possibly create, the pairing instability that leads to superconductivity? Simple model calculations that might shed light on this question are clearly of value, and in this paper we present such a study for a model Hamiltonian that we have considered in an attempt to hopefully mimic some of the physics of the stripe phases.

Previous work on the magnetic properties of the very weakly doped cuprates ${ }^{2}$ modeled the observed experimental support for stripe correlations ${ }^{3}$ using an effective Hamiltonian in which a (spatially) anisotropic exchange interaction was implemented to represent the stripe-induced magnetic energy scales. That is, in the direction parallel to the stripes the full local $\mathrm{Cu}-\mathrm{Cu}$ exchange would be present, while perpendicular to the stripes a reduced exchange would be encountered across such rivers of charge. Renormalized Hamiltonians of a similar simplifying spirit were also used in other studies of the doped cuprates. ${ }^{4-6}$

To this end, we have considered an effective Hamiltonian in which a carrier's ability to move along the river of charge is far larger than its freedom to move between the rivers. This reduced mobility reflects a carrier's lessened ability to move in an antiferromagnetically correlated region, such as exists between the rivers of charge. The simplest realization of such ideas corresponds to a model Hamiltonian with local magnetic exchange that is the same in all directions, but with a hopping parameter which is very different in one direction than the other. (Several studies of holes moving on two-leg ladders near half filling have considered such a problem, but all but one have employed a limit that is different to that which we have investigated. To be specific, these authors have considered the hopping between the chains of the ladder to be larger than that along each chain. ${ }^{7-10}$ The recent work of Weihong, et al., ${ }^{11}$ considers a limit similar to that of this paper, although their focus is two holes near half filling, not two electrons near zero filling.)

As a consequence of the above considerations, in this paper we consider the so-called $t-J-U$ model, which is defined by

$$
\begin{aligned}
H= & -\sum_{\langle i, j\rangle, \sigma} t_{i j}\left(c_{i, \sigma}^{\dagger} c_{j, \sigma}+\text { h.c. }\right)+\sum_{\langle i, j\rangle} J_{i j}\left(\mathbf{S}_{\mathbf{i}} \cdot \mathbf{S}_{\mathbf{j}}-\frac{1}{4} n_{i} n_{j}\right) \\
& +U \sum_{i} n_{i, \uparrow} n_{i, \downarrow},
\end{aligned}
$$

where the sites of a two-dimensional square lattice of size $L_{x} \times L_{y}$ with periodic boundary conditions are labeled by the indices $i$ and $j, t_{i j}$, and $J_{i j}$ are the hopping integrals and exchange couplings between sites $i$ and $j$, respectively, $c_{i, \sigma}$ is the annihilation operator for an electron of spin $\sigma, U$ is the on-site Hubbard repulsion energy, and $n_{i, \sigma}$ is the number operator for electrons at site $i$ with spin $\sigma$.

The most familiar strong-coupling variant of the Hubbard model is the $t-J$ model, and the physics of (square lattice) doped Mott insulators described by this model was reviewed by Dagotto. ${ }^{12}$ As emphasized by, e.g., Anderson, ${ }^{13}$ the vital component of the $t-J$ Hamiltonian is the constraint of no double occupancy. That is, in the $t-J$ model one does not use the electron creation and annihilation operators of Eq. (1), but rather one uses constrained creation and annihilation operators (for example, see the discussions in Ref. 12). However, the above $t-J-U$ Hamiltonian can be used to accomplish this same mathematical projection by taking $U \rightarrow \infty$ - this simplifying approach has been noted by a variety of researchers (see, e.g., Refs. 14-16), and will be used by us also.

In this paper we restrict $t_{i j}$ and $J_{i j}$ to be nonzero for near neighbors (NN) only. Further, we allow the hopping integral in the $x$ direction, $t_{x}$, to be different than the hopping integral in the $y$ direction, $t_{y}$. We have also investigated the 
physics that arises when $J_{x}$ is allowed to be different than $J_{y}$, but find that no qualitatively new physics arise as long as both $J_{x}$ and $J_{y}$ remain nonzero. Thus, from now on we set $J \equiv J_{x}=J_{y}$, and we analyze the resulting hopping anisotropy problem in terms of

$$
t_{x} \equiv t, \quad r=\frac{t_{y}}{t}
$$

By taking the limit $U / t \rightarrow \infty$ the only two (dimensionless) energy scales left in this problem are $J / t$ and $r$.

The physics of the $t-J-U$ model for isotropic NN hopping $(r=1)$ in the dilute electron density limit is clear: the antiferromagnetic exchange provides an attractive interaction between the electrons on neighboring sites forming a singlet state; however, a bound state that is formed from this interaction must do so in a manner which excludes double occupancy, a result that must follow in $U \rightarrow \infty$ limit. As mentioned above, this problem for isotropic hopping and exchange has already been studied elsewhere. ${ }^{14,15}$ Here we shall analyze the bound-state threshold exchange, to be denoted by $J_{c} / t$, as a function of hopping anisotropy $r$.

We consider the situation in which there are only two electrons present, in which case we can write the singlet wave function of a two-electron system as ${ }^{14,15}$

$$
\psi=\sum_{i, j} \phi(i, j) c_{i, \uparrow}^{\dagger} c_{j, \downarrow}^{\dagger}|0\rangle
$$

where $|0\rangle$ denotes the empty lattice. Any bound state can be solved for by the direct solution of the Schrödinger equation. Expressing $\phi(i, j)$ in terms of its Fourier transform, the latter of which involves the center of mass and relative momenta given by $\mathbf{Q}=\mathbf{k}_{\mathbf{1}}+\mathbf{k}_{\mathbf{2}}$ and $\mathbf{q}=\frac{1}{2}\left(\mathbf{k}_{\mathbf{1}}-\mathbf{k}_{\mathbf{2}}\right)$, with $\phi\left(\mathbf{k}_{\mathbf{1}}, \mathbf{k}_{\mathbf{2}}\right)$ $=\phi_{\mathbf{Q}}(\mathbf{q})$, where

$$
\phi\left(\mathbf{k}_{\mathbf{1}}, \mathbf{k}_{\mathbf{2}}\right)=\frac{1}{N} \sum_{i, j} e^{-i \mathbf{k}_{\mathbf{1}} \cdot \mathbf{r}_{i}-i \mathbf{k}_{\mathbf{2}} \cdot \mathbf{r}_{j}} \phi(i, j)
$$

with $N=L_{x} L_{y}$, the resulting equation for the bound-state energy $E$ and its associated wave function $\phi_{\mathbf{Q}}(\mathbf{q})$ is

$$
\phi_{\mathbf{Q}}(\mathbf{q})=\frac{\frac{U}{N} \sum_{\mathbf{k}} \phi_{\mathbf{Q}}(\mathbf{k})-\frac{1}{N} \sum_{\mathbf{k}} J(\mathbf{q}-\mathbf{k}) \phi_{\mathbf{Q}}(\mathbf{k})}{E-\varepsilon\left(\frac{\mathbf{Q}}{2}+\mathbf{q}\right)-\varepsilon\left(\frac{\mathbf{Q}}{2}-\mathbf{q}\right)} .
$$

The dispersions $\varepsilon(\mathbf{k})$ and $J(\mathbf{k})$, the former of which includes the hopping anisotropy, are given by $\varepsilon(\mathbf{k})=-2 t\left(\cos k_{x}\right.$ $\left.+r \cos k_{y}\right)$ and $J(\mathbf{k})=2 J\left(\cos k_{x}+\cos k_{y}\right)$.

The lowest-energy bound states for the $t-J-U$ model have zero center-of-mass momentum $(\mathbf{Q}=0)$, and a method of solution for these states was presented in the papers of $\mathrm{Lin}^{14}$ and Pethukov et al. ${ }^{15}$ However, in these papers the authors were considering a two-dimensional square plane with isotropic hopping, and thus were able to exploit $C_{x}=C_{y}$, where

$$
C_{x}=\frac{1}{N} \sum_{\mathbf{k}} \phi(\mathbf{k}) \cos \left(k_{x}\right) \quad C_{y}=\frac{1}{N} \sum_{\mathbf{k}} \phi(\mathbf{k}) \cos \left(k_{y}\right),
$$

a result that follows from the $s$-wave symmetry of the bound state. In our problem the Hamiltonian has a reduced symmetry, leading to $C_{x} \neq C_{y}$ (for $\left.r \neq 1\right)$, and thus we have employed a different approach that allows for us to identify the threshold exchange $J_{c} / t$ that leads to bound-state formation. This method, in application to this and other interesting problems (triangular, honeycomb, and kagome lattices) is explained elsewhere. $^{17}$

We find that the bound state for the anisotropic hopping problem is always symmetric under $\pi$ rotations, and is also symmetric under reflections along the $x$ or $y$ directions. That is, the ground state transforms under the identity representation of the rectangular point group, and thus continuously interpolates with the $s$-wave bound state for $r=1$. The threshold $J_{c} / t$ as a function of $r$ is given by the following expression:

$$
\frac{J_{c}}{t}=-\frac{16 \pi^{2} r^{5 / 2}}{g(r)}\left[f(r)-\sqrt{f(r)^{2}-\frac{g(r)}{64 \pi^{3} r^{3}}}\right],
$$

where $f$ and $g$ are functions given by

$$
f(r)=\frac{1}{32} \frac{\left[4\left(r^{3}-r^{2}+r-1\right) \sin ^{-1} \sqrt{\frac{r}{r+1}}-2 \pi r^{3}+4 r^{5 / 2}+2 \pi r^{2}+4 \sqrt{r}\right]}{\pi^{2} r^{5 / 2}}
$$

$$
g(r)=2\left(r^{2}-1\right) \sin ^{-1} \sqrt{\frac{r}{r+1}}-\pi r^{2}+2 r^{3 / 2}+2 \sqrt{r} .
$$

The resulting $J_{c} / t$ vs $r$ is plotted in Fig. 1 from which (i) the $r=1$ limit of $J_{c} / t=2$ result is found, confirming the known isotropic result, ${ }^{14,15}$ and (ii) the surprising result that as $r \rightarrow 0, J_{c} / t \rightarrow 0$. This latter result is important-it implies that one can form bound states for arbitrarily small $J / t$ if one is able to create a situation in which the hopping anisotropy is large enough. Further, it implies that for a fixed $J / t$, the binding energy of two electrons will become increasingly larger as this anisotropy is increased. We have plotted the bound-state energy vs $r$, for $J / t=2$ in Fig. 2, from which it is seen that a large hopping anisotropy can lead to remarkably large binding energies. While acknowledging our inability to relate this system directly to the high- $T_{c}$ systems, without further trepidation we evaluated the $r \rightarrow 0$ limit of the binding energy for $J / t=0.3$. We found a binding energy of 


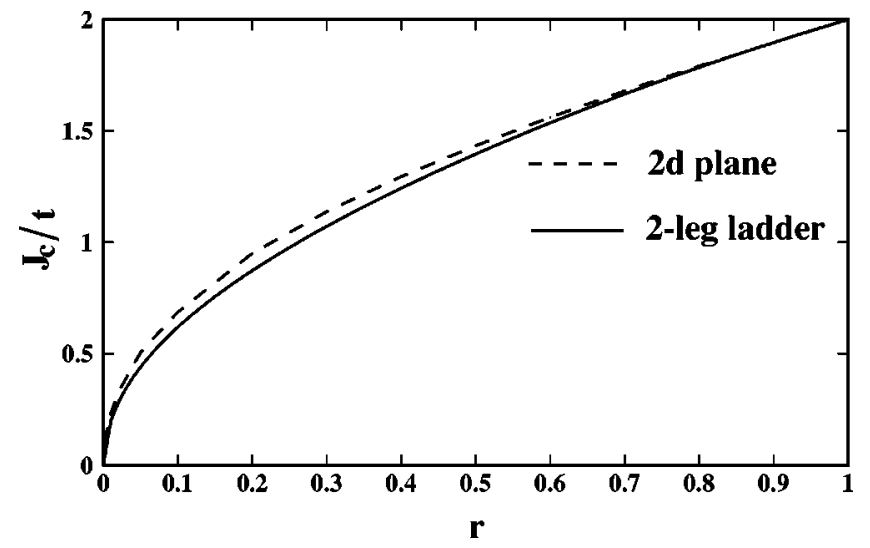

FIG. 1. The dashed line is a plot of Eq. (7), and shows how the threshold exchange for bound-state formation, $J_{c} / t$, varies as a function of hopping anisotropy, $r$, for an infinite two-dimensional plane. The solid line is a plot of Eq. (10), which is the same quantity for a two-leg ladder. Note the rapid decline to $J_{c} / t=0$ for both systems as $r \rightarrow 0$.

$0.0068 t$, and in units in which $t$ is roughly an $\mathrm{eV}$, this implies a binding energy of roughly $100 \mathrm{~K}$, a provocative energy.

The question remains, what is the nature of the correlations in such bound states? In an attempt to answer this question, we have solved the same problem as above not for an infinite two-dimensional plane, but for a two-leg ladder. Technically, this involves nothing more than using

$$
\sum_{\mathbf{q}} \rightarrow \frac{1}{2} \sum_{q_{y}=0, \pi} \frac{1}{2 \pi} \int_{-\pi}^{+\pi} d q_{x}
$$

in the various wave vector sums that appear in the evaluation of bound-state wave function and energy. The resulting $J_{c} / t$ as a function of $r$ is given by

$$
\frac{J_{c}}{t}=\frac{\left[(1+r)^{2}+2 \sqrt{r+r^{2}}-\sqrt{\left(1+r^{2}\right)^{2}+4 r}\right]}{r+1}
$$

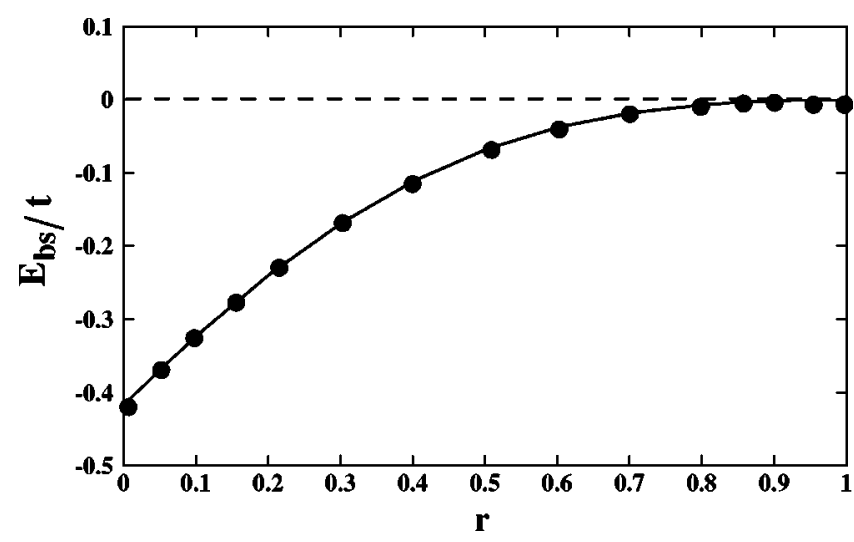

FIG. 2. The value of the bound-state energy, $E_{b s}$, relative to the bottom of the two-electron noninteracting band, the latter of which is equal to $-4 t(1+r)$, as a function of $r$ for $J / t=2$ for the infinite two-dimensional plane. For this value of $J / t$ a bound state is found for all $r$.

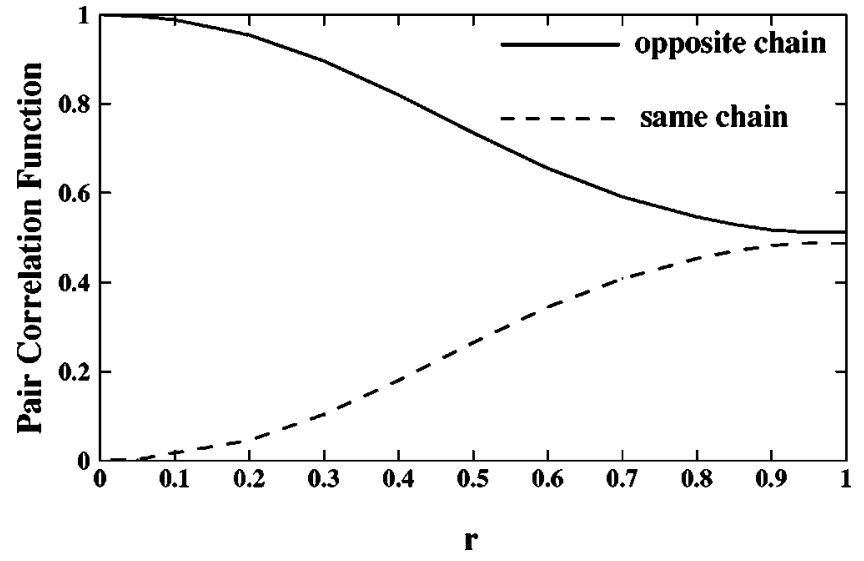

FIG. 3. The pair correlation for the bound state of the two-leg ladder evaluated for all electron positions, and then summed to yield the probabilities that the electrons are on the same chain or on opposite chains. This quantity is shown as a function of hopping anisotropy, $r$, and as in the previous figure, all data corresponds to $J / t=2$. Note that as $r \rightarrow 0$ the electrons are restricted to be on opposite chains only.

and is also shown in Fig. 1. Thus, we see that both the infinite two-dimensional plane and the infinitely long two-leg ladder behave in a similar fashion. Further, the nonanalytic behavior of $J_{c} / t$ is quantitatively similar in the extreme hopping anisotropy limit: in the limit of $r \rightarrow 0$ the twodimensional infinite plane has $J_{c} / t \sim(3 \pi / 4) \sqrt{r}$, while the two-leg ladder has $J_{c} / t \sim 2 \sqrt{r}$.

We have exploited this similarity by evaluating the paircorrelation function for the two-leg ladder, noting that the simpler geometry of the two-leg ladder more readily allows for us to understand the spatial character of the extreme hopping anisotropy bound states. More specifically, we have calculated the probability that the two electrons are on the same chain or are on opposite chains. Our results as a function of $r$ are shown in Fig. 3, from which it is seen that the character of the large hopping anisotropy (small $r$ ) bound states is two electrons moving on opposite chains, with the pairing resulting from a spin-exchange interaction between the chains (when the electrons are on neighboring sites).

This situation is qualitatively similar to two electrons moving in neighboring stripes that still experience an exchange coupling between the stripes due to the antiferromagnetically correlated domain between the stripes, but which have an effective hopping frequency that is much larger along the metallic rivers of charge than between such rivers. In this very simplistic picture, it seems that stripe correlations leading to parallel rivers of charge separated by antiferromagnetically correlated domains can (at least) enhance bound-state formation.

We have been able to examine the above systems analytically because of the relative simplicity of the zero-density two-electron problem. Of course, the natural question is: Does this result extrapolate to systems that are doped away from half filling? To address this question we have considered the problem of two holes in an otherwise half-filled $12 \times 2$ two-leg ladder for $J / t=0.3$. For all $r$ the ground state is $\mathbf{Q}=0$ and transforms under the identity representation of 
TABLE I. The two-hole binding energy, $E_{b}$, defined as $E_{b}$ $=E_{2 h}-2 E_{1 h}+E_{0 h}$, calculated for a $12 \times 2$ two-leg $t-J$ ladder with $J / t=0.3$, as a function of anisotropy parameter $r$.

\begin{tabular}{cc}
\hline \hline$r$ & $E_{b}$ \\
\hline 1.0 & -0.1189 \\
0.8 & -0.1614 \\
0.6 & -0.2224 \\
0.4 & -0.2803 \\
0.2 & -0.3137 \\
0.1 & -0.3254 \\
\hline \hline
\end{tabular}

the rectangular point group; however, we have evaluated the pairing correlation functions discussed in Ref. 10, and find that a $d$-wave like pairing symmetry is present for all $r$. The binding energy is again found to be substantially enhanced by the hopping anisotropy, a result previously noted in Ref.
11 - our data is listed below in Table I. Lastly, we again evaluated the probability that the holes existed on the same or opposite chains of the two-leg ladder, and our results are qualitatively similar to those of the two-electron problem that we have shown in Fig. 3 [the two-hole correlations near $r=1$ have a greater probability of being on the opposite chains (about 0.62), but otherwise these two systems behave in very similar ways].

Thus, the zero-density two-electron problem, and the two holes away from half-filling problem, behave in a very similar manner and are suggestive of the benefits of stripes in forming bound states. Whether or not this physics extrapolates to the pairing instabilities and, e.g., enhances the meanfield superconducting transition temperatures, is being investigated presently.

We thank David Johnston and Massimiliano Capezzali for many helpful comments, and Andrew Callan-Jones and Fred Nastos for assistance. This work was supported in part by the NSERC of Canada.
1 J.M. Tranquada, in Neutron Scattering in Layered Copper-Oxide Superconductors, edited by A. Furrer (Kluwer, Dordrecht, The Netherlands); J.M. Tranquada, Physica C 282-287, 166 (1997).

${ }^{2}$ A.H. Castro Neto and D. Hone, Phys. Rev. Lett. 76, 2165 (1996).

${ }^{3}$ F. Borsa et al., Phys. Rev. B 52, 7334 (1995).

${ }^{4}$ A. Aharony et al., Phys. Rev. Lett. 60, 1330 (1988).

${ }^{5}$ S. Doniach et al., Europhys. Lett. 6, 663 (1988).

${ }^{6}$ R.J. Gooding and A. Mailhot, Phys. Rev. B 48, 6132 (1993).

${ }^{7}$ T.M. Rice, S. Gopalan, and M. Sigrist, Europhys. Lett. 23, 45 (1993).

${ }^{8}$ R.M. Noack, S.R. White, and D.J. Scalapino, Physica C 270, 281
(1996).

${ }^{9}$ R.M. Noack et al., Phys. Rev. B 56, 7162 (1997).

${ }^{10}$ I. Bose and S. Gayen, J. Phys.: Condens. Matter 11, 6427 (1999).

${ }^{11}$ Z. Weihong et al., cond-mat/0007388 (unpublished).

${ }^{12}$ E. Dagotto, Rev. Mod. Phys. 66, 763 (1994).

${ }^{13}$ P.W. Anderson, Adv. Phys. 46, 3 (1997).

${ }^{14}$ H.Q. Lin, Phys. Rev. B 44, 4674 (1991).

${ }^{15}$ A.G. Petuhkov, J. Galán, and J. Verǵes, Phys. Rev. B 46, 6212 (1992).

${ }^{16}$ M. Kagan and T.M. Rice, J. Phys. C 6, 3771 (1994).

17 S. Basu and R. J. Gooding (unpublished). 